\title{
Validación predictiva de un método de clasificación funcional en adultos mayores
}

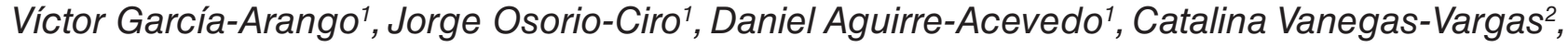 \\ Carmen Clavijo-Usuga ${ }^{2}$ y Jaime Gallo-Villegas ${ }^{1}$
}

Forma de citar

García-Arango V, Osorio-Ciro J, Aguirre-Acevedo D, Vanegas-Vargas C, Clavijo-Usuga C, Gallo-Villegas J. Validación pre-dictiva de un método de clasificación funcional en adultos mayores. Rev Panam Salud Publica. 2021;45:e15.

https://doi.org/10.26633/RPSP.2021.15

RESUMEN

Objetivo. Evaluar la validez predictiva de un método de clasificación funcional (CF) sobre el uso de los servicios de urgencias y hospitalización, mortalidad y costos de la atención en salud en adultos mayores.

Métodos. Estudio de cohorte retrospectivo que incluyó 2168 adultos mayores en un programa de atención de las enfermedades crónicas no transmisibles (ECNT) en Medellín (Colombia). Los pacientes fueron estratificados según un método de CF con base en el estado funcional, presencia de factores de riesgo y control de la comorbilidad. Durante un año de seguimiento, se evaluó la validez predictiva de la CF sobre los desenlaces estudiados; se midieron la discriminación y la calibración con el estadístico-C y de Hosmer-Lemeshow (H-L), respectivamente.

Resultados. El promedio de edad fue 74,6 \pm 7,9 años; el 40,8\% ( $n=884)$ fueron hombres y 7,7\% $(n=168)$ murieron. El riesgo de muerte (razón de posibilidades [OR, por su sigla en inglés]: 1,767; 3,411; 8,525), hospitalización (OR: 1,397; 2,172; 3,540) y un costo elevado de la atención en salud (OR: 1,703; 2,369; 5,073) aumentaron en la medida que hubo un deterioro en la CF, clases 2B, 3 y 4, respectivamente. El modelo predictivo para el desenlace muerte mostró una buena capacidad de discriminación (estadístico-C $=0,721$ ) y calibración (estadístico de $\mathrm{H}-\mathrm{L}=10,200 ; P=0,251$ ).

Conclusión. Existe una relación de dosis y respuesta entre el deterioro de la CF y un riesgo más elevado de muerte, hospitalización y costo elevado. La CF tiene validez predictiva para la tasa de mortalidad y podría utilizarse para la estratificación de adultos mayores en programas de atención de las ECNT con miras a dirigir las acciones de intervención.

Palabras clave Fragilidad; envejecimiento; enfermedad crónica; urgencias médicas; hospitalización; mortalidad; costos de la atención en salud.

Diversos datos demográficos de América Latina y en particular de Colombia, muestran que la población se encuentra en una "transición epidemiológica" (1). Esta transición epidemiológica se caracteriza por un aumento de la expectativa de vida y una disminución de la mortalidad, lo que lleva a un incremento de la población de adultos mayores y, en consecuencia, de la frecuencia de enfermedades crónicas no transmisibles (ECNT) y los costos de la atención $(2,3)$.
El uso de los servicios de urgencias y hospitalización son marcadores de costo elevado (4), reconocidos ampliamente como posibles indicadores del rendimiento de la atención médica (4). En los últimos años, se han desarrollado modelos predictivos de la demanda de los servicios de urgencias y hospitalización (5-7); estas herramientas son valiosas debido a que permiten identificar personas con riesgo elevado de desenlaces adversos (5-7). Estos modelos utilizaron, en su mayoría, datos

\footnotetext{
1 Universidad de Antioquia, Medellín, Colombia. $\bowtie$ Jaime Gallo-Villegas, jaime.gallo@udea.edu.co
}

2 Institución Prestadora de Servicios de Salud Universitaria, Medellín, Colombia. 
administrativos, debido al acceso y recolección fáciles (8-11); no obstante, la inclusión de información de otras fuentes, como la salud autorreportada o el estado funcional, mejora su capacidad predictiva $(7,12-14)$.

Por otro lado, se sabe que un peor desempeño en pruebas físicas, como la fuerza de agarre y la velocidad de la marcha, se asocian con morbilidad y mortalidad más elevadas en adultos mayores $(15,16)$. Esta asociación se puede explicar al deterioro funcional durante el envejecimiento, subyace a la presencia de enfermedad clínica manifiesta y no detectada, y a la exposición a factores de riesgo (inactividad física, tabaquismo, entre otros) para mortalidad prematura a lo largo de la vida (17-20).

Hasta la fecha, según el conocimiento de los autores, no se han publicado modelos predictivos latinoamericanos que hayan incluido información relacionada con los diagnósticos previos, datos suministrados de forma directa por el paciente, mediciones objetivas del estado funcional y la presencia de fragilidad, para identificar individuos con necesidades en salud. La importancia de estas investigaciones radica en la posibilidad de identificar subgrupos de adultos mayores con un riesgo más elevado de desenlaces adversos (10, 20-22). Estas herramientas podrían contribuir en la implementación de intervenciones específicas estratificadas según el riesgo basal al interior de los programas de atención de los adultos mayores con ECNT.

En época reciente, se desarrolló un método de clasificación funcional para estratificar adultos mayores que ingresaron a un programa de atención de pacientes con ECNT, con miras a hacer una intervención diferencial según las necesidades. En este método de clasificación, se incluyeron variables relacionadas con el estado funcional (apoyo monopodal, velocidad de la marcha y fragilidad) que mostraron capacidad predictiva independiente de desenlaces adversos en adultos mayores (15, $16,19)$. No obstante, se desconoce la validez predictiva de este método de clasificación funcional cuando dichas variables se incorporan de manera conjunta.

Como hipótesis, se planteó que el método de clasificación funcional propuesto tiene validez predictiva, y que un deterioro en la clasificación funcional se relaciona con un incremento en el uso de los servicios de urgencias y hospitalización, mayor riesgo de mortalidad y costos elevados en la atención en salud. El objetivo de este estudio fue evaluar la validez predictiva de un método de clasificación basado en el estado funcional, la presencia de factores de riesgo y el control de la comorbilidad, sobre el uso de los servicios de urgencias y hospitalización, mortalidad y costos de la atención en salud en adultos mayores.

\section{MATERIALES Y MÉTODOS}

Se realizó un estudio de cohorte retrospectivo para el desarrollo y validación de un modelo predictivo tipo 2a según la guía Transparent Reporting of a multivariable prediction model for Individual Prognosis or Diagnosis (TRIPOD, por sus sigas en inglés) (23). En este estudio se incluyeron 2168 adultos mayores afiliados a la Entidad Promotora de Salud (EPS) "Nueva EPS", que ingresaron al programa de atención de pacientes con ECNT llamado "Ser más" de una Institución Prestadora de Servicios de Salud (IPS Universitaria), y que vivían en el área metropolitana del Valle de Aburrá cerca al centro de atención, en Medellín, Colombia.

\section{Sujetos}

Los criterios de inclusión fueron: hombres y mujeres, con edad mayor o igual a 60 años, residentes en el área metropolitana y que ingresaron al programa de atención entre agosto de 2017 y enero de 2018.

Los criterios de exclusión fueron los adultos mayores en quienes no fue posible realizar la clasificación funcional al ingreso al programa de atención o se retiraron antes de un año de seguimiento por razones diferentes a los desenlaces evaluados.

\section{Predictores}

Los sujetos fueron estratificados según un método de clasificación funcional, con base en la evaluación de tres componentes, así: i) el estado funcional, a partir de la prueba de apoyo monopodal (24), la medición de la velocidad de la marcha en cinco metros (25) y el índice de fragilidad de Gröningen (26); ii) la presencia de factores de riesgo como obesidad, bajo peso con un porcentaje elevado de grasa corporal, tabaquismo, hipotimia (27) y mala condición física (28); y iii) la presencia de comorbilidad no controlada por la presencia de diabetes mellitus, hipertensión arterial, insuficiencia renal, enfermedad pulmonar obstructiva crónica (EPOC), enfermedad coronaria, enfermedad cerebrovascular, valvulopatía cardíaca e insuficiencia cardíaca, según las guías actuales (29-33).

Con los tres componentes descritos se obtuvieron cinco categorías posibles: i) clase 1 , estado funcional conservado con comorbilidad controlada; ii) clase $2 \mathrm{~A}$, estado funcional conservado con comorbilidad controlada, pero con presencia de factores de riesgo; iii) clase $2 \mathrm{~B}$, estado funcional conservado con comorbilidad no controlada; iv) clase 3, estado funcional alterado con comorbilidad no controlada; y v) clase 4 , estado funcional perdido con comorbilidad no controlada.

Se consideró estado funcional perdido si el tiempo en la prueba de apoyo monopodal fue menor de $10 \mathrm{~s}(24)$, la velocidad de la marcha en cinco metros fue menor de $0,6 \mathrm{~m} / \mathrm{s}$ (25) y el índice de fragilidad de Gröningen fue igual o mayor a 4 puntos (26).

La presencia de obesidad se definió por un índice de masa corporal (IMC) mayor de 30, bajo peso con un porcentaje elevado de grasa corporal si el IMC fue menor de 18,5 y la sumatoria de tres pliegues cutáneos de grasa (tricipital, abdominal y muslo medio) fue mayor de $60 \mathrm{~mm}$, hipotimia si la pregunta sobre depresión en el bienestar subjetivo fue calificada mayor de 4 (27) y mala condición física si el consumo de oxígeno máximo estimado fue menor de 7 unidades de la tasa metabólica basal (TMB) (28).

También, además de la clasificación funcional, se incluyeron la edad y el sexo como otros predictores.

\section{Desenlaces}

El uso del servicio de urgencias se definió si el paciente tuvo tres o más visitas $(5,6)$, mientras que el uso del servicio de hospitalización se consideró si el paciente fue hospitalizado una o más veces durante un año de seguimiento $(5,7)$. Para el desenlace muerte se incluyeron todas las causas. Un gerente de sistemas de información en salud evaluó estos desenlaces a partir del sistema Gestión Hospitalaría IPS (GHIPS) de la IPS Universitaria y de las bases de datos asistenciales que recopilan 
información demográfica, clínica, ingresos y defunciones de los afiliados.

Un costo elevado de la atención en salud se definió si el paciente estaba por encima del percentil 90 de los costos totales de la atención en salud (costo elevado) durante el año de seguimiento $(4,5,7)$. El costeo fue realizado por un economista experto, perteneciente a la Unidad de Costos y Presupuestos de la IPS Universitaria, quien cuantificó los costos totales de las prestaciones que requirió cada paciente durante el período de seguimiento. Se utilizó la metodología de costeo total (full costing) (34), y de costeo basado en actividades (ABC, por sus siglas en inglés) (34), que permite asignar los costos directos e indirectos según las prestaciones requeridas en los servicios hospitalarios (hospitalización, cirugía, unidad de cuidados especiales, unidad de cuidados intensivos y recurso humano) y ambulatorios (consulta externa, medicamentos, ayudas diagnósticas y recurso humano).

Las personas encargadas de evaluar los desenlaces estuvieron cegadas de la clasificación funcional de los pacientes.

\section{Análisis estadístico}

Al inicio, se realizó un análisis exploratorio de los datos. Se utilizó el promedio y la desviación estándar o la mediana y el rango intecuartil según el supuesto de distribución normal para las variables cuantitativas, y la frecuencia y la proporción para las variables categóricas.

Se compararon las curvas de supervivencia de Kaplan-Meier según la clasificación funcional con la prueba de logaritmo del rango (log-Rank test). Se utilizó un modelo de riesgos proporcionales de Cox para estimar el riesgo de cocientes instantáneos (HR, por sus siglas en inglés) del desenlace de mortalidad, y un modelo de regresión logística binaria, para estimar la razón de posibilidades (OR, por sus siglas en inglés) para los desenlaces uso de los servicios de urgencias y hospitalización, y los costos de la atención en salud, ajustados por la edad y el sexo.

Se dividió la muestra de manera aleatoria en dos bases de datos; en una de las muestras, se desarrolló el modelo de regresión logística binaria para todos los desenlaces, mientras que en la otra, se evaluó el rendimiento predictivo a partir de una validación interna (23). Se utilizó el estadístico-C como medida de discriminación, y el estadístico de Hosmer-Lemeshow (H-L) como medida de calibración del modelo desarrollado que incluyó la clasificación funcional, la edad y el sexo para la presentación de los diferentes desenlaces de interés.

Para todos los análisis se presentó el intervalo de confianza del 95\% (IC95\%). Los análisis se realizaron con el programa STATA $^{\circledR}$ versión 14.0 (StataCopr LP, College Station, Texas, Estados Unidos) y el programa IBM SPSS ${ }^{\circledR}$ Statistics, versión 21.0 (IBM, New York, Estados Unidos).

El estudio fue aprobado por el Comité de Ética de la IPS Universitaria. El registro de cada paciente en la base de datos fue nombrado con un código que impidió su identificación. Se tuvo en cuenta las normas para la investigación en salud del Ministerio de la Protección Social de Colombia en la Resolución 008430 de 1993 y los principios de la declaración de Helsinki (35).

\section{RESULTADOS}

De las 5105 personas que ingresaron al programa de atención de pacientes con ECNT, 2168 cumplieron los criterios de
FIGURA 1. Flujograma que muestra el proceso de inclusión de los participantes al estudio.

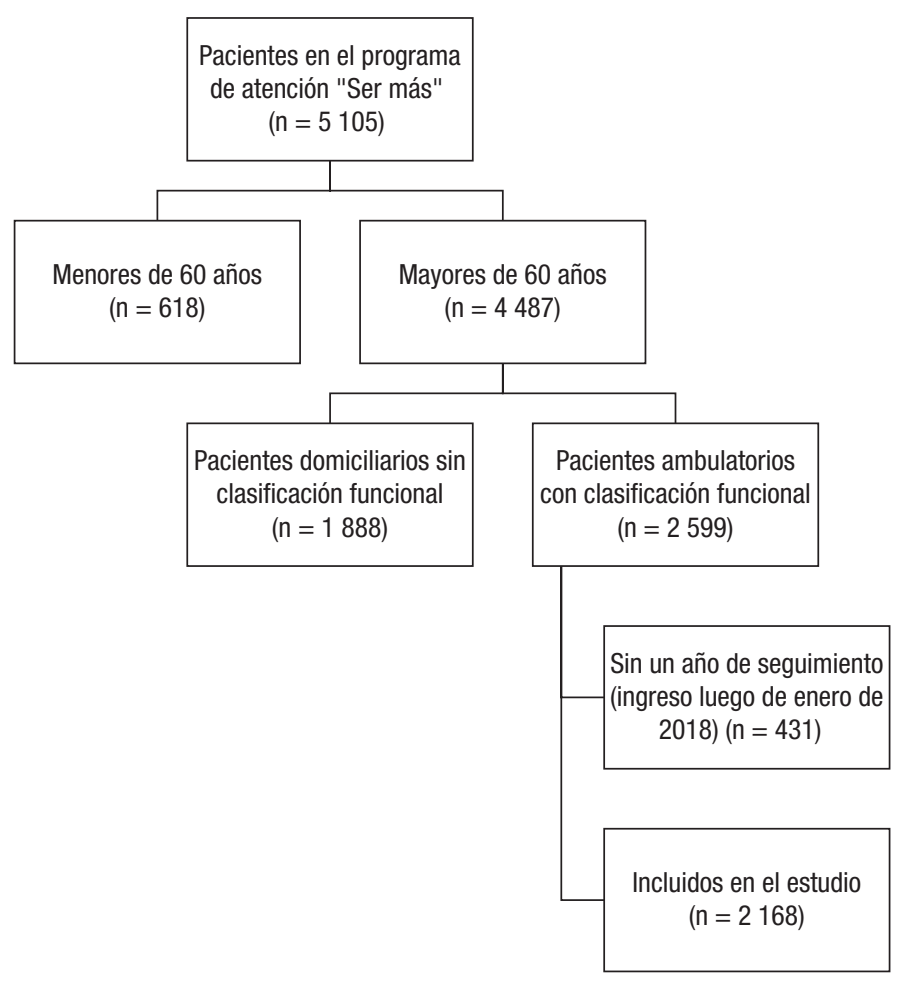

Elaboración propia a partir de los resultados presentados.

inclusión y no tenían criterios exclusión; por tal razón, fueron incluidas en el estudio (figura 1).

Entre los participantes, se encontró un promedio de edad de $74,6 \pm 7,9$ años, de índice de masa corporal de 28,3 $\pm 5,3$, y de consumo de oxígeno máximo estimado de 5,1 $\pm 2,1$ TMB; 40,8\% $(n=884)$ eran hombres y el $72,6 \%(n=1532)$ de los pacientes presentaba sobrepeso $\mathrm{u}$ obesidad. La hipertensión fue la comorbilidad más frecuente, con 92,3\% ( $n=2000)$, seguida de la diabetes mellitus con $49,2 \%(n=1067)$ (cuadro 1$)$. Del total de la muestra, $6,7 \%(n=145)$ visitaron el servicio de urgencias tres o más veces; $31,8 \%(n=690)$ tuvieron una o más hospitalizaciones; $7,7 \%(\mathrm{n}=168)$ murieron, y 215 pacientes fueron clasificados por encima del percentil 90 de los costos de atención (cuadro 1).

Debido a que los pacientes pertenecientes a la clase funcional 1 (estado funcional conservado con comorbilidad controlada) solo fueron 17 personas, y ninguno de ellos presentó los desenlaces uso del servicio de urgencias, mortalidad y costo elevado, se tomó la decisión de utilizar como categoría de referencia la clase funcional 2A (estado funcional conservado con comorbilidad controlada, pero con presencia de factores de riesgo) para los análisis (cuadros 1 y 2).

En la figura 2 se presentan las curvas de supervivencia según la clasificación funcional. Las curvas presentan diferencias importantes donde hay mayor probabilidad acumulada de supervivencia en las clases $1,2 \mathrm{~A}$ y $2 \mathrm{~B}$ ( $\chi^{2}$ de la prueba log-Rank $=182,947 ; P<0,001)$. En los pacientes de la clase 4, se aprecia una disminución en la probabilidad de supervivencia antes de los 100 días (3,3 meses) (figura 2).

Acorde a las curvas de supervivencia, el riesgo es cada vez más elevado a medida que la clase funcional aumenta. 
CUADRO 1. Descripción de las características demográficas, antropométricas, clínicas, funcionales y de laboratorio en toda la muestra y según el uso de los servicios de urgencias y hospitalización, muerte y costo elevado de la atención en salud $(\mathrm{n}=2168)$

\begin{tabular}{|c|c|c|c|c|c|}
\hline Características & Urgencias & Hospitalización & Mortalidad & Costo elevado & $\begin{array}{l}\text { Todos los } \\
\text { pacientes incluidos } \\
\text { en el estudio }\end{array}$ \\
\hline n (\%) & $145(6,7)$ & $690(31,8)$ & $168(7,7)$ & $215(9,9)$ & $2168(100)$ \\
\hline Edad en años, media (DE) & $75,4(7,6)$ & $75,7(7,9)$ & $78,9(7,8)$ & $74,6(7,0)$ & $74,6(7,9)$ \\
\hline Hombres, n (\%) & $63(43,4)$ & $302(43,8)$ & $80(47,6)$ & $96(44,7)$ & $884(40,8)$ \\
\hline \multicolumn{6}{|l|}{ Antropométricas } \\
\hline Talla en cm, media (DE) & $154,9(9,0)$ & $154,9(9,4)$ & $155,9(9,5)$ & $155,3(9,7)$ & $155,0(9,2)$ \\
\hline IMC en $\mathrm{kg} / \mathrm{m}^{2}$, media (DE) & $27,6(5,6)$ & $28,2(5,9)$ & $26,1(5,8)$ & $28,7(6,4)$ & $28,3(5,3)$ \\
\hline$\sum$ pliegues cutáneos de grasa en cm, media (DE) & $70,3(31,7)$ & $74,4(35,9)$ & $67,0(33,6)$ & $75,5(37,5)$ & $76,7(34,9)$ \\
\hline \multicolumn{6}{|l|}{ Clínicas } \\
\hline PAS en mmHg, media (DE) & $133,7(24,7)$ & $132,4(23,4)$ & $129,8(24,4)$ & $133,9(25,4)$ & $132,3(21,0)$ \\
\hline PAD en mmHg, media (DE) & $74,0(16,8)$ & $73,2(14,0)$ & $72,6(16,5)$ & $74,2(16,0)$ & $73,8(12,5)$ \\
\hline \multicolumn{6}{|l|}{ Funcionales } \\
\hline $\mathrm{VO}_{2}$ máx (TMB), media (DE) & $5,0(1,9)$ & $5,1(2,1)$ & $5,2(1,9)$ & $5,0(1,9)$ & $5,1(2,1)$ \\
\hline Índice de fragilidad, mediana (RI) & $3,0(2,0-4,0)$ & $3,0(2,0-4,0)$ & $4,0(3,0-6,0)$ & $3,0(2,0-4,0)$ & $3,0(2,0-4,0)$ \\
\hline Apoyo monopodal en segundos, mediana (RI) & $3,0(1,0-11,2)$ & $3,0(1,0-12,0)$ & $2,0(1,0-6,2)$ & $2,0(1,0-10,0)$ & $8,0(1,0-17,0)$ \\
\hline $\begin{array}{l}\text { Velocidad de la marcha en } 5 \mathrm{~m} \text { en segundos, } \\
\text { mediana (RI) }\end{array}$ & $0,8(0,7-0,8)$ & $0,8(0,7-0,8)$ & $0,7(0,7-0,8)$ & $0,8(0,7-0,8)$ & $0,8(0,7-1,0)$ \\
\hline \multicolumn{6}{|l|}{ Clasificación funcional } \\
\hline Clase funcional $1, \mathrm{n}(\%)^{\mathrm{a}}$ & $0(0,0)$ & $4(0,6)$ & $0(0,0)$ & $0(0,0)$ & $17(0,8)$ \\
\hline Clase funcional $2 \mathrm{~A}, \mathrm{n}(\%)^{\mathrm{b}}$ & $48(33,1)$ & $232(33,6)$ & $36(21,4)$ & $60(27,9)$ & $902(41,6)$ \\
\hline Clase funcional $2 \mathrm{~B}, \mathrm{n}(\%)^{\mathrm{c}}$ & $63(43,4)$ & $321(46,5)$ & $70(41,7)$ & $106(49,3)$ & $979(45,2)$ \\
\hline Clase funcional $3, \mathrm{n}(\%)^{\mathrm{d}}$ & $23(15,9)$ & $75(10,9)$ & $25(14,9)$ & $24(11,2)$ & $169(7,8)$ \\
\hline C-LDL en mg/dL, media (DE) & $87,0(40)$ & $87,2(36,2)$ & $86,7(38,4)$ & $88,9(36,6)$ & $87,2(36,1)$ \\
\hline C-HDL en mg/dL, media (DE) & $49,4(14,9)$ & $48,1(13,9)$ & $45,9(15,1)$ & $48,9(15,5)$ & $48,3(13,2)$ \\
\hline Triglicéridos en mg/dL, media (DE) & $158,6(100,2)$ & $148,0(88,9)$ & $150,0(100,5)$ & $151,4(101,4)$ & $151,2(92,4)$ \\
\hline Creatinina en mg/dL, media (DE) & $1,2(0,7)$ & $1,1(0,6)$ & $1,3(0,9)$ & $1,1(0,6)$ & $1,0(0,4)$ \\
\hline TFG en mL/min, media (DE) & $50,6(22,8)$ & $51,7(22,5)$ & $45,6(24,2)$ & $51,6(21,5)$ & $55,2(22,9)$ \\
\hline
\end{tabular}

aClase funcional 1: estado funcional conservado, con comorbilidad controlada.

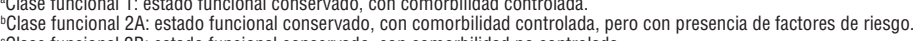

"Clase funcional 2B: estado funcional conservado, con comorbilidad no controlada.

Clase funcional 3: estado funcional alterado, con comorbilidad no controlada

DE, desviación estándar; IMC, índice de masa corporal; PAS, presión arterial sistólica; PAD, presión arterial diastólica; FC, frecuencia cardíaca; Ipm, latidos por minuto; V0 máx consumo de oxígeno máximo; TMB, tasa metabólica basal; $\mathrm{RI}$, rango intercuartílico; EPOC, enfermedad pulmonar obstructiva crónica; HbA1c, hemoglobina glucosilada A1c; C-LDL, colesterol de la lipoproteína de baja densidad; C-HDL, Colesterol de la lipoproteína de alta densidad; TFG, tasa de

filtración glomerular.

Cuadro de elaboración propia a partir de los resultados presentados.

En comparación con los pacientes de la clase 2A, aquellos clasificados como 2B presentaron un $\mathrm{HR}=1,767$ (IC95\%: $1,182-2,642 ; P=0,006) ;$ los de clase 3, un $\mathrm{HR}=3,411$ (IC95\%: 2,041-5,700; $P=0,001$ ), y los de clase 4 , un HR = 8,525 (IC95\%: 5,281-13,761; $P=0,001$ ) luego de ajustar por la edad y el sexo (cuadro 2). Este mismo efecto dosis-respuesta se observó para el riesgo de hospitalización y costo elevado en la atención en salud (cuadro 2). Con relación al uso del servicio de urgencias, se encontró un riesgo más elevado para la clase 3 (OR: 2,741; IC95\%: 1,611-4,663; $P=0,001)$ y la clase 4 (OR: 2,115; IC95\%: 1,044-4,283; $P=0,037$ ) (cuadro 2).

Con respecto a la validez predictiva, solo para el desenlace mortalidad, el modelo de regresión logística binario desarrollado mostró simultáneamente una buena capacidad de discriminación (estadístico-C =0,720; IC95\%: 0,660-0,780) y calibración (estadístico $\mathrm{H}-\mathrm{L}=10,200 ; P=0,251$ ) (figura 3). Asî 
CUADRO 2. Magnitud de la asociación de la clasificación funcional, la edad y el sexo, con la mortalidad (modelo de riesgos proporcionales de Cox), uso de los servicios de urgencias y hospitalización, y costo elevado de la atención en salud (modelos de regresión logística) para toda la muestra $(n=2151)$

\begin{tabular}{|c|c|c|c|c|c|c|c|c|}
\hline \multirow{3}{*}{ Variables } & \multicolumn{2}{|c|}{ Urgencias } & \multicolumn{2}{|c|}{ Hospitalización } & \multicolumn{2}{|c|}{ Mortalidad } & \multicolumn{2}{|c|}{ Costo elevado } \\
\hline & \multicolumn{2}{|c|}{$R^{2}=0,015$} & \multicolumn{2}{|c|}{$R^{2}=0,025$} & \multicolumn{2}{|c|}{$R^{2}=0,108$} & \multicolumn{2}{|c|}{$R^{2}=0,027$} \\
\hline & $\mathrm{OR}$ & (IC 95\%) & $\mathrm{OR}$ & (IC 95\%) & $\mathrm{HR}$ & (IC 95\%) & $\mathrm{OR}$ & (IC 95\%) \\
\hline Hombres & 1,106 & $(0,785-1,558)$ & 1,196 & $(0,992-1,441)$ & 1,327 & $(0,98-1,798)$ & 0,829 & $(0,622-1,104)$ \\
\hline Clase funcional $2 \mathrm{~A}$ & \multicolumn{2}{|c|}{ Grupo de referencia } & \multicolumn{2}{|c|}{ Grupo de referencia } & \multicolumn{2}{|c|}{ Grupo de referencia } & \multicolumn{2}{|c|}{ Grupo de referencia } \\
\hline Clase funcional 2B & 1,219 & $(0,828-1,795)$ & 1,397 & $(1,143-1,708)$ & 1,767 & $(1,182-2,642)$ & 1,703 & $(1,224-2,371)$ \\
\hline
\end{tabular}

$\mathrm{R}^{2}$, seudo $\mathrm{R}$ cuadrado de McFadden; $\mathrm{OR}$, razón de posibilidades (por sus siglas en inglés); HR, riesgo de cocientes instantáneos (por sus siglas en inglés).

¿Estado funcional conservado, con comorbilidad controlada, pero con presencia de factores de riesgo.

Estado funcional conservado, con comorbilidad no controlada.

Estado funcional alterado, con comorbilidad no controlada.

Estado funcional perdido, con comorbilidad no controlada. Los modelos fueron ajustados por edad y sexo.

Cuadro de elaboración propia a partir de los resultados presentados.

FIGURA 2. Curvas de Kaplan-Meier que muestran las diferencias en la distribución de las probabilidades de supervivencia según la clasificación funcional.

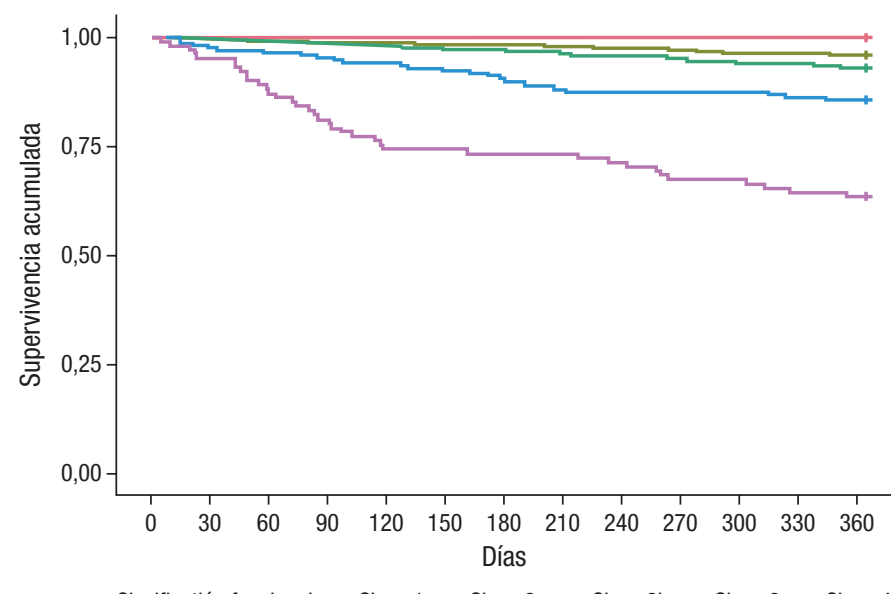

Elaboración propia a partir de los resultados presentados.

mismo, este modelo fue el que tuvo un mayor $\mathrm{R}^{2}$ de McFadden $(0,108)$.

Con base en la validez del modelo, se desarrolló la siguiente ecuación para estimar la probabilidad de muerte a un año a partir de la clasificación funcional, la edad y el sexo:

$$
p(\text { muerte })=\frac{1}{1+e^{-(-8,213+0,058 \times(\text { edad en añas })+0,283 \text { (hombre })+\beta_{3} \text { (clasificación funcional) }}}
$$

\section{Donde}

$P$ es la probabilidad de muerte a un año, condicionada a la edad de la persona, sexo y clasificación funcional; $\beta_{3}=0,838$ si es clase $2 B ; \beta_{3}=1,462$ si es clase $3 ; y \beta_{3}=2,596$ si es clase 4 . Las categorías de referencia fueron: para sexo (sexo $=0$ si es mujer) y clasificación funcional (clasificación funcional $=0 \mathrm{si}$ es clase 2A). Por ejemplo, para una persona con 75 años, hombre, y de clasificación funcional 3, la probabilidad de muerte a un año es $10,8 \%$. Si este paciente tuviera una clasificación funcional 4, la probabilidad de muerte a un año asciende a $27,3 \%$.

Para los demás desenlaces, como el uso de los servicios de urgencias y hospitalización, y costo elevado en salud, la capacidad de discriminación fue baja (estadístico-C $=0,570$ [IC95\%: 0,500-0,640]; 0,609 [IC95\%: 0,570-0,650]; y 0,632 [IC95\%: $0,570-0,690]$, respectivamente). También, para los desenlaces uso de los servicios de urgencias (estadístico H-L = 14,740; $P=0,065$ ) y hospitalización (estadístico $\mathrm{H}-\mathrm{L}=16,820 ; P=0,032$ ), la calibración no fue buena (figura 3). El desenlace costo elevado mostró una buena calibración (estadístico H-L $=11,420$; $P=0,179)$ (figura 3).

\section{DISCUSIÓN}

Entre los principales hallazgos de este estudio se encuentran: i) en la medida que hay un deterioro en la clasificación funcional, aumenta el riesgo (dosis-respuesta) de muerte, hospitalización y costo elevado en la atención en salud; ii) se observaron diferencias en las probabilidades de supervivencia según las categorías de la clasificación funcional; iii) el modelo predictivo que incluyó la clasificación funcional, la edad y el sexo para el desenlace muerte es válido debido a su capacidad de discriminación y calibración; y iv) no obstante, los modelos desarrollados para los otros desenlaces (uso de los servicios de urgencias y hospitalización, y costo elevado en la atención en salud), no son válidos.

\section{Clasificación funcional y mortalidad}

El deterioro en la clasificación funcional aumenta el riesgo de muerte de una forma dosis-respuesta, y tiene validez predictiva; un hallazgo similar al reportado en otras investigaciones $(15,16)$. En el metaanálisis realizado por Cooper y cols., encontraron que una disminución de la fuerza de agarre, la velocidad de la marcha y el pararse y sentarse en una silla, se asocia con un riesgo más elevado de muerte en adultos mayores (15). De igual forma, Nofuji y cols., encontraron que una disminución de la fuerza de agarre, velocidad de la marcha, y tiempo de estancia 
FIGURA 3. Calibración de los modelos de regresión logística desarrollados para los desenlaces uso de los servicios de urgencias y hospitalización, mortalidad y costo elevado de la atención en salud ( $n=1080)$.
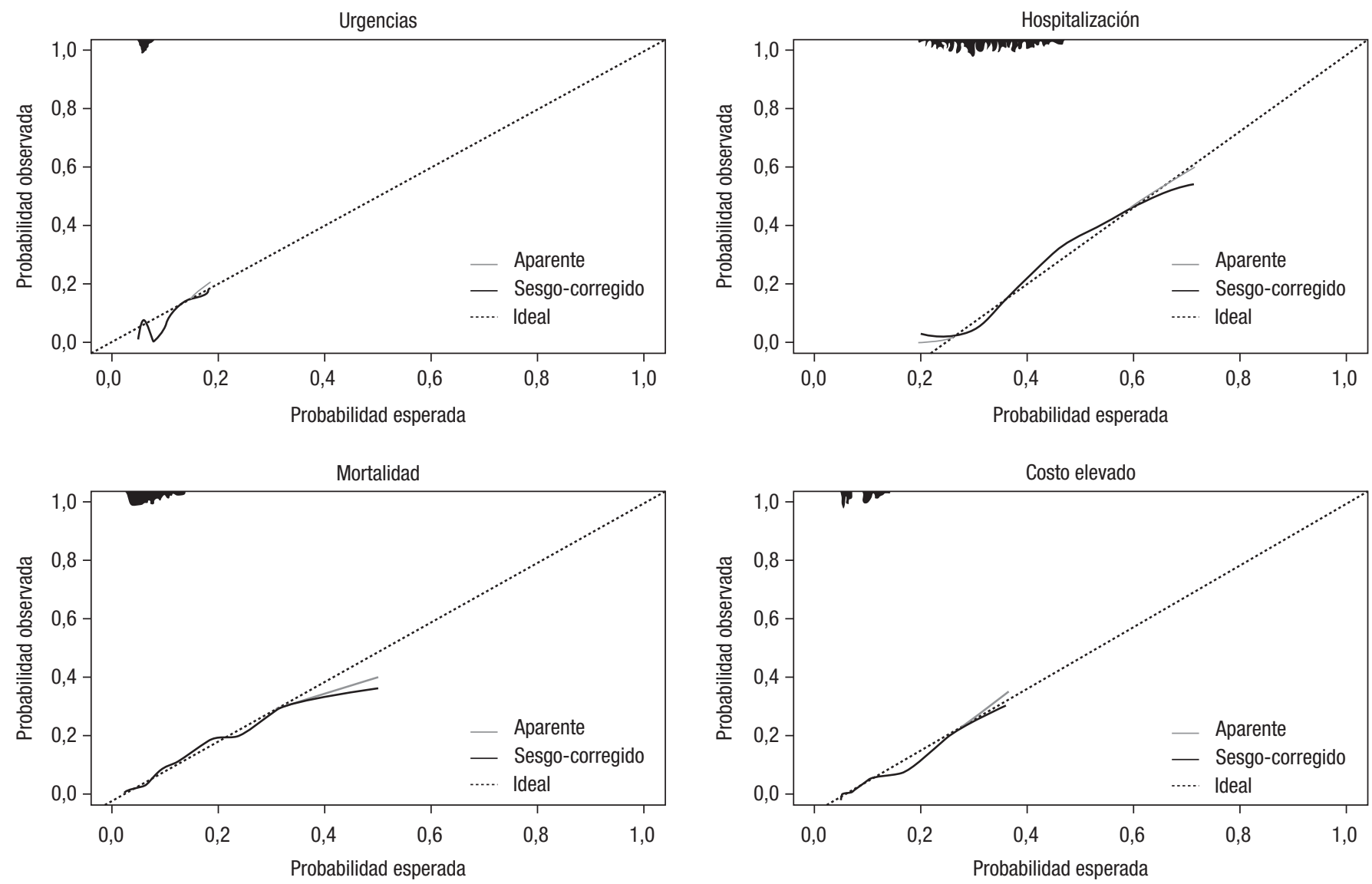

Figura de elaboración propia a partir de los resultados presentados.

monopodal, se asocia con un riesgo más elevado de muerte por todas las causas y muerte de origen cardiovascular (16).

Los resultados de esta investigación, y otros trabajos $(15,16)$, tal vez puedan explicarse por la relación que existe entre la función y la exposición de factores de riesgo a lo largo de la vida, la presencia de ECNT manifiestas y no detectadas, y la velocidad del proceso de envejecimiento (17-19). También, es importante resaltar que el estado funcional en etapas tardías de la vida está determinado no solo por su tasa de declinación desde la vida media, si no también por el pico alcanzado durante la fase de crecimiento y desarrollo $(18,19)$. La relación entre la clasificación funcional y la mortalidad podría expresar diferencias en el desarrollo intrauterino y posnatal temprano entre los individuos, lo cual influye tanto en el estado funcional como en la supervivencia $(18,19)$. Estos resultados muestran la importancia de evaluar el estado funcional de los adultos mayores, con herramientas de fácil aplicación en la rutina diaria, y a un costo bajo, con el fin de predecir el riesgo de muerte en programas de atención de pacientes con ECNT.

\section{Clasificación funcional, uso de los servicios de salud y costo elevado}

El deterioro en la clasificación funcional aumenta el riesgo del uso de los servicios de urgencias y hospitalización, y costo elevado en la atención en salud, un hallazgo similar al reportado

por otros autores (5-7). No obstante, en los modelos predictivos desarrollados, la capacidad de discriminación de la clasificación funcional para el uso de los servicios de urgencias y hospitalización, y costo elevado de la atención en salud, fue menor a la reportada en la literatura por otros investigadores (5-7). Por su parte, Leininger y cols. encontraron que la capacidad de discriminación de un modelo básico con variables sociodemográficas se incrementa (para el uso del servicio de urgencias y un costo elevado) cuando se agrega la valoración de las necesidades en salud autorreportadas (presencia de comorbilidades, hábitos, prescripción de medicamentos, acceso a los servicios de salud) (5).

Por otro lado, Wherry y cols. encontraron que la capacidad de discriminación de un modelo básico con variables sociodemográficas aumenta (para el uso de los servicios de urgencias y hospitalización, y costo elevado) cuando se agregan principalmente tres dominios de la salud autorreportada (demanda previa de servicios de salud, condiciones de salud y calidad de vida) (6).

Así mismo, el estudio de Perrin y cols., reportaron que la capacidad de discriminación de un modelo basado en datos administrativos se incrementa (para el uso del servicio de hospitalización y costo elevado) cuando se agregan las respuestas a tres preguntas del autorreporte del estado de salud y funcional (puntaje general de la salud autorreportado, necesidad de ayuda en una o más actividades de la vida diaria y la presencia de comorbilidades) (7). 
Las diferencias observadas entre estos resultados y los hallazgos de otros investigadores (5-7) se pueden explicar por la inclusión de múltiples variables independientes en los modelos de esos estudios, y no la agregación de información en una clasificación funcional, como lo propusimos en este trabajo. También, es importante resaltar que, a diferencia de este estudio, en las investigaciones previas (5-7) solo se evaluó la capacidad de discriminación y no la calibración, lo cual limita la validez predictiva de dichos modelos.

A diferencia de lo observado con el uso del servicio de hospitalización y costo elevado, la clasificación funcional no tuvo una relación dosis-respuesta con el uso del servicio de urgencias. El riesgo del uso del servicio de urgencias fue mayor en la clase 3 que en la clase 4 . Esto puede explicarse en parte, debido a que una vez los pacientes fueron identificados como clase 4, recibieron una atención domiciliaria, tal vez requirieron menos visitas al servicio de urgencias, lo cual llevó a un subregistro de dicho desenlace. También, es probable que el punto de corte utilizado (tres o más visitas) para definir la presencia del uso del servicio de urgencias $(5,6)$, haya influido en los resultados.

Los resultados de este trabajo, y de otras investigaciones, plantean la importancia de combinar tanto información administrativa, como aquella relacionada con la evaluación del estado de salud y funcional, con el fin de mejorar el rendimiento predictivo de los modelos con relación al uso de los servicios de urgencias y hospitalización, y costo elevado de la atención en salud. Es posible que exista información relevante en otros predictores no incluidos en este estudio que, al no estar correlacionados entre sí, pueden producir una mejoría aditiva incremental en el rendimiento predictivo.

\section{Limitaciones}

El presente estudio tienen algunas limitaciones, que se describen a continuación: i) la recolección de los datos fue retrospectiva; no obstante, se hizo un cegamiento de la clasificación funcional para quienes estuvieron encargados de obtener los desenlaces; ii) la posibilidad de generalización de los resultados aplica para adultos mayores en el contexto de programas de atención de pacientes con ECNT; por tal razón, se requieren estudios de validación externa en otros contextos clínicos y países latinoamericanos; iii) es importante mencionar que, de acuerdo a la clasificación funcional, los pacientes recibieron una intervención estratificada en un programa de atención que incluyó actividad física y estrategias de educación grupal; sin embargo, el efecto de dicha intervención pudo ir en contra de la hipótesis de estudio; iv) también, un seguimiento más estrecho por parte del médico general y el médico internista, según la clasificación funcional, podría haber llevado a una mejoría en la condición clínica de cada sujeto, un objetivo perseguido por el programa de atención, y que a su vez pudo influir en la presentación de los desenlaces; y v) es posible que la clasificación funcional haya cambiado en el tiempo.

\section{CONCLUSIÓN}

Existe una relación dosis-respuesta entre el deterioro de la CF y un riesgo más elevado de muerte, hospitalización y costo elevado. La CF tiene validez predictiva para mortalidad y podría utilizarse para la estratificación de adultos mayores en programas de atención de las ECNT con miras a dirigir las acciones de intervención.

Contribución de los autores. VGA, JGV y DAA concibieron el estudio original. VGA, JOC, CVV y CCU participaron en la recolección de los datos. Todos los autores contribuyeron en el análisis de los datos e interpretación de los resultados. VGA y JGV escribieron el primer borrador del manuscrito. Todos los autores revisaron y aprobaron la versión final del documento.

Agradecimientos. Los autores agradecen a Ángela Jaramillo y Ana Rivillas, de la Unidad de Investigación IPS Universitaria; a Lethzer Vásquez y Alejandro Aristizábal, de la Unidad de Costos y Presupuestos IPS Universitaria, y a Daniel a Cardona y Michele Guarín, gerentes en Sistemas de Información, Facultad Nacional de Salud Pública, Universidad de Antioquia.

\section{Conflictos de interés. Ninguno declarado por los autores.}

Financiación. Esta investigación se realizó con recursos de la IPS Universitaria y la Universidad de Antioquia, Medellín, Colombia. Los patrocinadores no participaron de ninguna manera en el diseño del estudio, la colecta y análisis de los datos, la decisión de publicar este trabajo ni la preparación del manuscrito.

Declaración. Las opiniones expresadas en este manuscrito son únicamente responsabilidad de los autores y no reflejan necesariamente los de la Revista Panamericana de Salud Pública o la Organización Panamericana de la Salud.

\section{REFERENCIAS}

1. Matus-López M. Thinking about long-term care policies for Latin America. Salud Colect. 2015;11(4):485-96.

2. Mercer AJ. Updating the epidemiological transition model. Epidemiol Infect. 2018;146(6):680-7.

3. Kalseth J, Halvorsen T. Health and care service utilisation and cost over the life-span: a descriptive analysis of population data. BMC Health Serv Res. 2020;20(1):435.

4. Wammes JJG, van der Wees PJ, Tanke MAC, Westert GP, Jeurissen PPT. Systematic review of high-cost patients' characteristics and healthcare utilisation. BMJ Open. 2018;8(9):e023113.

5. Leininger LJ, Friedsam D, Voskuil K, DeLeire T. Predicting highneed cases among new Medicaid enrollees. Am J Manag Care. 2014;20(9):e399-407.
6. Wherry LR, Burns ME, Leininger LJ. Using self-reported health measures to predict high-need cases among Medicaid-eligible adults. Health Serv Res. 2014;49 (S2):2147-72.

7. Perrin NA, Stiefel M, Mosen DM, Bauck A, Shuster E, Dirks EM. Selfreported health and functional status information improves prediction of inpatient admissions and costs. Am J Manag Care. 2011;17(12):e472-8.

8. Meenan RT, Goodman MJ, Fishman PA, Hornbrook MC, O'Keeffe-Rosetti MC, Bachman DJ. Using risk-adjustment models to identify high-cost risks. Med Care. 2003;41(11):1301-12.

9. Coderch J, Sánchez-Pérez I, Ibern P, Carreras M, Pérez-Berruezo X, Inoriza JM. Predicción del riesgo individual de alto coste sanitario para la identificación de pacientes crónicos complejos. Gac Sanit. 2014;28(4):292-300. 
10. Ng SH, Rahman N, Ang IYH, Sridharan S, Ramachandran S, Wang $\mathrm{DD}$, et al. Characterization of high healthcare utilizer groups using administrative data from an electronic medical record database. BMC Health Serv Res. 2019;19(1):452.

11. Labby D, Wright B, Broffman L, Holtorf M. Drivers of highcost medical complexity in a medicaid population. Med Care. 2020;58(3):208-15.

12. DeSalvo KB, Bloser N, Reynolds K, He J, Muntner P. Mortality prediction with a single general self-rated health question: a meta-analysis. J Gen Intern Med. 2006;21(3):267-75.

13. DeSalvo KB, Jones TM, Peabody J, McDonald J, Fihn S, Fan V, et al. Health care expenditure prediction with a single item, self-rated health measure. Med Care. 2009;47(4):440-7.

14. Fleishman JA, Cohen JW. Using information on clinical conditions to predict high-cost patients. Health Serv Res. 2010;45(2):532-52.

15. Cooper R, Kuh D, Hardy R, Mortality Review G, Falcon, Teams HAS. Objectively measured physical capability levels and mortality: systematic review and meta-analysis. BMJ. 2010;341:c4467.

16. Nofuji Y, Shinkai S, Taniguchi Y, Amano H, Nishi M, Murayama H, et al. Associations of walking speed, grip strength, and standing balance with total and cause-specific mortality in a general population of Japanese elders. J Am Med Dir Assoc. 2016;17(2):184 e1-7.

17. Seals DR, Melov S. Translational geroscience: emphasizing function to achieve optimal longevity. Aging (Albany NY). 2014;6(9):718-30.

18. Kuh D, Karunananthan S, Bergman H, Cooper R. A life-course approach to healthy ageing: maintaining physical capability. Proc Nutr Soc. 2014;73(2):237-48.

19. Kuh D, New Dynamics of Ageing Preparatory N. A life course approach to healthy aging, frailty, and capability. J Gerontol. 2007;62(7):717-21.

20. Rijken M, van der Heide I. Identifying subgroups of persons with multimorbidity based on their needs for care and support. BMC Fam Pract. 2019;20(1):179.

21. Smeets RGM, Elissen AMJ, Kroese M, Hameleers N, Ruwaard D. Identifying subgroups of high-need, high-cost, chronically ill patients in primary care: A latent class analysis. PLoS One. 2020;15(1):e0228103.

22. Anderson M, Revie CW, Stryhn H, Neudorf C, Rosehart Y, Li W, et al. Defining 'actionable' high-cost health care use: results using the Canadian Institute for Health Information population grouping methodology. Int J Equity Health. 2019;18(1):171.

23. Moons KG, Altman DG, Reitsma JB, Ioannidis JP, Macaskill P, Steyerberg EW, et al. Transparent Reporting of a multivariable prediction model for Individual Prognosis or Diagnosis (TRIPOD): explanation and elaboration. Ann Intern Med. 2015;162(1):W1-73.

24. Kuh D, Bassey EJ, Butterworth S, Hardy R, Wadsworth ME, Musculoskeletal Study T. Grip strength, postural control, and functional leg power in a representative cohort of British men and women: associations with physical activity, health status, and socioeconomic conditions. J Gerontol. 2005;60(2):224-31.
25. Studenski S, Perera S, Patel K, Rosano C, Faulkner K, Inzitari M, et al. Gait speed and survival in older adults. JAMA. 2011;305(1):50-8.

26. Steverink N, Slaets J, Schuurmans H, van Lis M. Measuring frailty: Development and testing of the Groningen Frailty Indicator (GFI). Gerontologist. 2001;41(special issue 1):236-7.

27. Organización para la Cooperación y el Desarrollo Económicos. Guidelines on measuring subjective well-being. París: OECD Publishing; 2013. Disponible en: http://dx.doi.org/10.1787/9789264191655-en Acceso el 4 de diciembre de 2020.

28. Jurca R, Jackson AS, LaMonte MJ, Morrow JR, Jr., Blair SN, Wareham $\mathrm{NJ}$, et al. Assessing cardiorespiratory fitness without performing exercise testing. Am J Prev Med. 2005;29(3):185-93.

29. Davies MJ, D'Alessio DA, Fradkin J, Kernan WN, Mathieu C, Mingrone $\mathrm{G}$, et al. Management of hyperglycemia in type 2 diabetes, 2018: a consensus report by the American Diabetes Association (ADA) and the European Association for the Study of Diabetes (EASD). Diabetes Care. 2018;41(12):2669-701.

30. Williams B, Mancia G, Spiering W, Agabiti Rosei E, Azizi M, Burnier M, et al. 2018 ESC/ESH Guidelines for the management of arterial hypertension. Eur Heart J. 2018;39(33):3021-104.

31. Task Force M, Montalescot G, Sechtem U, Achenbach S, Andreotti F, Arden C, et al. 2013 ESC guidelines on the management of stable coronary artery disease: the Task Force on the management of stable coronary artery disease of the European Society of Cardiology. Eur Heart J. 2013;34(38):2949-3003.

32. Ponikowski P, Voors AA, Anker SD, Bueno H, Cleland JGF, Coats AJS, et al. 2016 ESC Guidelines for the diagnosis and treatment of acute and chronic heart failure: The Task Force for the diagnosis and treatment of acute and chronic heart failure of the European Society of Cardiology (ESC) Developed with the special contribution of the Heart Failure Association (HFA) of the ESC. Eur Heart J. 2016;37(27):2129-200.

33. Burkes RM, Donohue JF. An update on the Global Initiative for Chronic Obstructive Lung Disease 2017 Guidelines with a focus on classification and management of stable COPD. Respir Care. 2018;63(6):749-58.

34. Spacirova Z, Epstein D, Garcia-Mochon L, Rovira J, Olry de Labry Lima A, Espin J. A general framework for classifying costing methods for economic evaluation of health care. Eur J Health Econ. 2020;21(4):529-42.

35. Schuklenk U. Helsinki Declaration revisions. Issues Med Ethics. 2001;9(1):29.

Manuscrito recibido el 30 de junio de 2020. Aceptado para su publicación, tras revisión, el 14 de julio de 2020. 


\section{Predictive validity of a functional classification method in older adults}

ABSTRACT Objective. Evaluate the predictive validity of a functional classification (FC) method for the use of emergency services and hospitalization, mortality, and health care costs among older adults.

Methods. Retrospective cohort study that included 2168 older adults in a chronic noncommunicable disease (CNCD) care program in Medellin, Colombia. Patients were stratified according to a FC method based on functional status, presence of risk factors, and control of comorbidity. During one year of follow-up, the predictive validity of the FC method was assessed for the studied outcomes. Discrimination and calibration were measured with the C-statistic and Hosmer-Lemeshow (HL) test, respectively.

Results. The average age was $74.6 \pm 7.9$ years; $40.8 \%(n=884)$ were men and $7.7 \%(n=168)$ died. The risk of death (odds ratio [OR]: 1.767; 3.411; 8.525), hospitalization (OR: 1.397; 2.172; 3.540) and high cost of health care (OR: $1.703 ; 2.369 ; 5.073$ ) increased in proportion to a deterioration in functional classification (classes 2B, 3, and 4, respectively). The predictive model for the outcome of death showed good capacity for discrimination (C-statistic $=0.721$ ) and calibration (HL statistic 10.200; $P=0.251)$.

Conclusion. There is a dose-response relationship between deterioration in $\mathrm{FC}$ and a higher risk of death, hospitalization, and high cost. FC has predictive validity for the mortality rate and could be used to stratify older adults in CNCD care programs with a view to guiding interventions.

Keywords Frailty; aging; chronic disease; emergencies; hospitalization; mortality; health care costs.

\section{Validação preditiva de método de classificação funcional em idosos}

RESUMO Objetivo. Avaliar a validade preditiva de um método de classificação funcional (CF) para a utilização de serviços de emergência e internação hospitalar, mortalidade e custos da atenção de saúde em idosos.

Métodos. Estudo de coorte retrospectivo com 2168 idosos atendidos em um programa de atenção de doenças crônicas não transmissíveis (DCNT) em Medellín, Colômbia. Um método de CF foi usado para estratificar os participantes segundo o estado funcional, presença de fatores de risco e controle de comorbidades. No período de acompanhamento de um ano, a validade preditiva da CF foi avaliada para os desfechos de interesse. A capacidade discriminatória (estatística C) e a calibração (teste de Hosmer-Lemeshow [H-L]) do modelo foram avaliadas.

Resultados. A média de idade dos participantes do estudo foi $74,6 \pm 7,9$ anos, 40,8\% ( $n=884$ ) eram do sexo masculino e $7,7 \%(n=168)$ vieram a óbito. Houve aumento do risco de óbito (odds ratio [OR] 1,767; 3,411-8,525), internação hospitalar (OR 1,397; 2,172-3,540) e custo elevado da atenção de saúde (OR 1,703; 2,369-5,073) com o declínio funcional - classes funcionais 2B, 3 e 4, respectivamente. O modelo preditivo para o desfecho de óbito demonstrou boa capacidade discriminatória (estatística $C=0,721$ ) e calibração (estatística $\mathrm{H}-\mathrm{L}=10,200 ; P=0,251$ ).

Conclusão. Há uma relação de dose-resposta entre o declínio da CF e risco maior de óbito, internação hospitalar e custo elevado da atenção. A CF tem validade preditiva para a taxa de mortalidade e poderia ser utilizada na estratificação de idosos em programas de atenção de DCNT para ajudar a direcionar as medidas de intervenção.

Palavras-chave Fragilidade; envelhecimento; doença crônica; emergências; hospitalização; mortalidade; custos de cuidados de saúde. 